\title{
CONGENITAL PYLORIC STENOSIS
}

Together with Reports of Two Cases of Duodenal and One Case of Jejunal Atresia

By David Levi, M.S., F.R.C.S.

The aetiology of congenital pyloric stenosis remains unknown. The theory at present fashionable is that the development of the neuro-muscular plexus has been delayed and that the pyloric sphincter fails to relax and consequently hypertrophies. The fact is that the whole musculature of the stomach is hypertrophic. This hypertrophy is not present at birth, but appears with considerable rapidity in patients presenting symptoms. The following case history shows that the hypertrophy is acquired rather than congenital:-

The author was asked to operate on a child aged ten days, both of whose brothers had been operated on by him for pyloric stenosis. The mother and the maternity nurse were certain that this child had the same trouble. No tumour was found on examination or at operation and the stomach appeared to be perfectly normal. The abdomen was therefore closed without further procedure. Three weeks later the abdomen was re-opened on account of persistent symptoms and on this occasion a well-formed tyumour was present. The muscle was therefore divided in the usual manner and the child made a good recovery.

Babies suffering from pyloric stenosis are now brought to hospital comparatively early in the course of the disease. The starved infants of former years are now rarely seen. We hope that more children are breast-fed. The truth of the saying that "breast milk never disagrees with a baby' is generally recognized. The teaching that 'if a breast-fed baby vomits persistently, it probably has pyloric stenosis-look for it' is bearing fruit.

The disease occurs more frequently in males and usually manifests itself in infants between three and eight weeks old. It may commence earlier. One child in this series vomited from birth. If an infant with pyloric stenosis reaches the age of three months without operation, the condition is said to undergo spontaneous resolution. This is not absolutely true as operation was necessary for one child of five and a half months (Harris, C., 1938, Proc. Roy. Soc. Med., 31, 767). The babies are always hungry; alert children and the condition may occur in several members of the same family. As already indicated, the author has operated on three brothers successively in the same family.

\section{Pathology}

The stomach shows a generalized hypertrophy of the whole musculature reaching an extreme o degree at the pylorus. The transition from the iु thickened muscle of the stomach to the thin duodenum is abrupt. The circular fibres of the

pyloric sphincter are most affected.
Viewed from the duodenal side, the hypertrophic phincter has been likened to a small $\vec{c}$ cervix, but it does not project into the duodenum? as the cervix projects into the vagina. The fold of duodenal mucosa at the junction of the duodenum has been called the dangerous angle and may $\overrightarrow{b e c}$ injured at operation.

With the persistent vomiting and failure of foôd to reach the small intestine, the blood chemistry is seriously altered. The blood urea rises, the alkali reserve is increased, the urine is diminished in quantity and its chlorides diminished or absent. $\frac{\mathrm{D}}{\mathrm{Q}}$ The alkalosis is caused by the persistent loss of acid $\stackrel{2}{\vec{T}}$ radicles in the vomit, whilst the blood urea is raised by the combination of starvation and vomiting.

In a child which has died from the disease the stomach is hypertrophic, the hypertrophy extending up to the oesophageal opening. It is most:marked at the pylorus. The gastric mucosa shows 3 . changes caused by the associated gastritis. There may be ' coffee ground ' content inside the organ. The body generally shows signs of dehydration, alkalosis and starvation. Apart from the effects of these pathological and biochemical changes the body and organs are normal.

\section{Symptoms and Signs}

The first symptom is vomiting. This is com- N monly projectile and comes on after feeding. The vomit never contains bile. It persists and is associated with constipation and loss of weight. In the later stages the vomit may contain blood coming from the inflamed gastric mucosa. If a newborn child vomits and the vomit is bile-stained, the child is not suffering from pyloric stenosis but $\stackrel{\mathbb{D}}{\mathbb{P}}$ may be suffering from intestinal obstruction. The $\frac{\odot}{\mathbb{Q}}$ 
obstruction may be in the duodenum, as in duodenal atresia, or anywhere down the intestinal . tract as far as the anus. New-born babies may vomit because of a tight anus, not necessarily a complete atresia; or there may be some other cause for the vomiting.

To examine such a child the physician should wear a mask to prevent cross-infection, and should have warm hands. The child should be in a warm room and a sterile feed should be available as well as a sterile finger stall. The abdomen is uncovered and inspected. Often if the sterile finger stall is put on the index finger of the right hand and this is placed in the baby's mouth the firm pyloric tumour will be felt mid-way between the ensiform and the umbilicus, just to the right of the mid-line. Marked segmentation of the rectus abdominis, the edge of the liver, or the right kidney may give misleading evidence. The act of sucking stimulates gastric peristalsis; the pylorus will contract and become palpable. The tumour becomes hard and soft. If a tumour is felt, the diagnosis is assured. If the finger stall method is not successful a feed should be given. Whilst the child is drinking, the abdomen is palpated and the tumour should become palpable. As the stomach fills, peristalsis may be seen passing from left to right; often the whole outline of the stomach is visible. Gastric peristalsis may be visible in children who are not suffering from pyloric stenosis and by itself is of no diagnostic value. Associated with a palpable tumour, however, it clinches the diagnosis of pyloric obstruction. Finally, the child may vomit in characteristic fashion at the end of the feed. Should there still be doubt about the diagnosis, the stomach should be aspirated before each feed. A persistent gastric residue is suggestive of pyloric stenosis.

Help may also be obtained by an X-ray examination. Infants take a barium meal quite well; it can be given by spoon feeding or can be run into the stomach through a catheter. As barium is apt to lodge and remain in the colon becoming hard and inspissated and even giving rise to obstructive symptoms, however, it is seldom used in the investigation of such infants. A lipiodol meal is preferable.

A large stomach with gastric delay is the usual finding, although occasionally an opaque meal will pass the pylorus in these infants. Sometimes it is possible to demonstrate the long thin pyloric canal running through the sphincter. The stomach is often very mobile and large peristaltic waves can be seen passing along it. In most cases it should be possible to make the diagnosis on the history and examination without recourse to the $\mathrm{X}$-ray department.

Should the child be neglected, it will vomit persistently. It will become alkalosed, having lost many of its chloride radicles in the vomit. Later the vomit may contain blood coming from the inflamed gastric mucosa. The blood urea rises and one case under the author's care had a blood urea of $98 \mathrm{mg}$. per $100 \mathrm{cc}$. of blood. It made a good recovery after operation and is now 14 years old.

The advanced stages of the disease are now fortunately rarely seen. The picture is unmistakable. The infant is emaciated, bluish grey in colour, with bright eyes and loose skin which hangs in folds from the abdomen and thighs, and it is constipated to an extreme degree.

\section{Treatment}

The treatment of hypertrophic pyloric stenosis is surgical, provided that there is an efficient surgical team and correct nursing and hospital facilities. The place for medical treatment in pyloric stenosis is at the onset of symptoms when the diagnosis is still in doubt, or when efficient surgery is not obtainable. Once the diagnosis is made, valuable time should not be wasted in medical measures; the surgeon should be consulted at once.

There are three components to the surgical team. The special hospital, the nursing staff and the surgeon and house officers. For perfectoo results the three must work together. The hospital $\risingdotseq$ should have separate rooms for nursing mothers and their babies. If the baby is bottle-fed the child must have a room to itself. Everything that goes into the room must be sterile. All visitors, attendants and maids must be free from colds and their throats must be free from haemolytic streptococci. Children must not have soiled napkins changed at feeding times. The nurse changing the child must regard this operation in the same way as she would regard a surgical dressing. She must scrub up before and afterwards. The soiled napkins must be put into a covered sterilized receptacle and sent away. The soiled squares must not all be tipped into an open dustbin kept under the kitchen sink as was the custom in a certain hospital 25 years ago.

The only necessary pre-operative treatment is a gastric lavage to empty the stomach of its retained contents and air. It is not the author's routine practice to give pre-operative fluids by other routes than the mouth. No absolutely dogmatic statement can be made. If a child is grossly dehydrated, intravenous fluids are given preoperatively.

Donovan (1946, Ann. Surg., 708, I5) states that pre-operative preparation is the greatest factor in bringing the mortality to its present level and given ' $80 \mathrm{ml}$. of physiologic salt solution with or 
without dissolved glucose twice a day under the skin.' This would be equivalent to giving $\mathrm{I} \frac{1}{2} \mathrm{pt}$. of fluid subcutaneously to an adult, and as can be imagined would be by no means conducive to comfort. The fluid would be better taken by mouth. By efficient surgery, infants suffering from pyloric stenosis are enabled to do this.

Gastric lavage should be carried out a quarter of an hour before operation. The nurse should make sure that the stomach is emptied of air as well as fluid. If the child is not disturbed by the catheter, it may be left in place during the operation, to enable any air swallowed subsequently to be extracted. One minim of tincture of opium may be given if the child is at all restless.

\section{Operation}

The temperature of the operating theatre should be as near to $80^{\circ} \mathrm{F}$. as possible. If necessary; the child may be placed on the operating table on two partially filled rubber hot water bottles. The temperature of the water in the bottles should be tested with a thermometer and should be $98.4^{\circ}$. Local anaesthesia is invariably used and is produced by means of $\frac{1}{2}$ per cent. ethocaine with or without adrenaline.

A general anaesthetic is neither necessary nor desirable. The child is less distressed by the procedure and is able to take fluid by the mouth earlier after local anaesthesia.' Never more than Io $\mathrm{ml}$. of the anaesthetic are required; 2 to $3 \mathrm{ml}$. are placed subcutaneously along the line of the proposed incision. A little less than this is divided between the muscles on either side. The remaining 2 to $3 \mathrm{ml}$. are placed deep to the linea alba in the extra-peritoneal space. The whole procedure can be carried out through one or two skin punctures.

A mid-line incision is found to be most satisfactory. Nothing should be allowed to leave the abdomen other than the hypertrophic pyloric tumour. This can be delivered by the use of two pairs of plain dissecting forceps. It is not necessary for the operator to put any part of the hand into the peritoneal cavity. The pylorus is held between the finger and thumb and is squeezed gently until it becomes hard. A line is chosen on its anterior surface as free from blood vessels as possible and the peritoneum is incised from the duodenum to beyond the hypertrophic sphincter. The muscle is then split with a blunt dissector or the end of a blunt-ended pair of scissors and the mucosa is seen to bulge through. Closure of the abdomen is surprisingly easy as the peritoneum comes together without tension. Gentleness is more important than speed. With local anaesthesia and a warm theatre, speed is of little moment.

The essentials of after-treatment consist of the gradual resumption of normal feeding with special care to guard against the risk of gastroenteritis. If the child has been recently taken from ${ }_{c}$ the breast, every endeavour is made to re-establish

\section{Pyloric Stenosis Post-Operative Feeding}

I. Breast Feeding. For the first two days expressed mother's millk is used. The child is not put to the breasto until two to three days after operation, as its condition? allows. Feeds commence $3 \frac{1}{2}$ hours after operation.

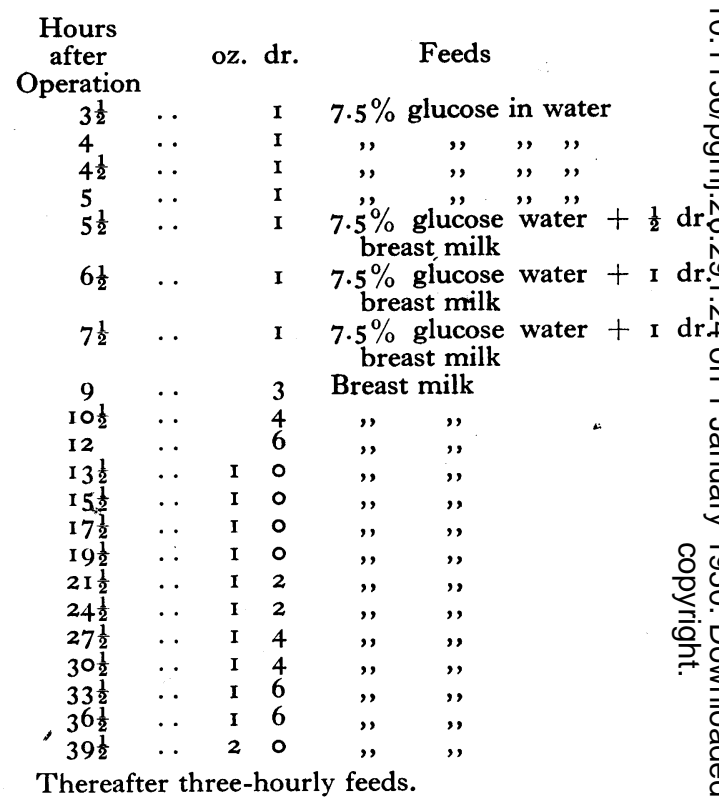

\section{Bottle Feeding.}

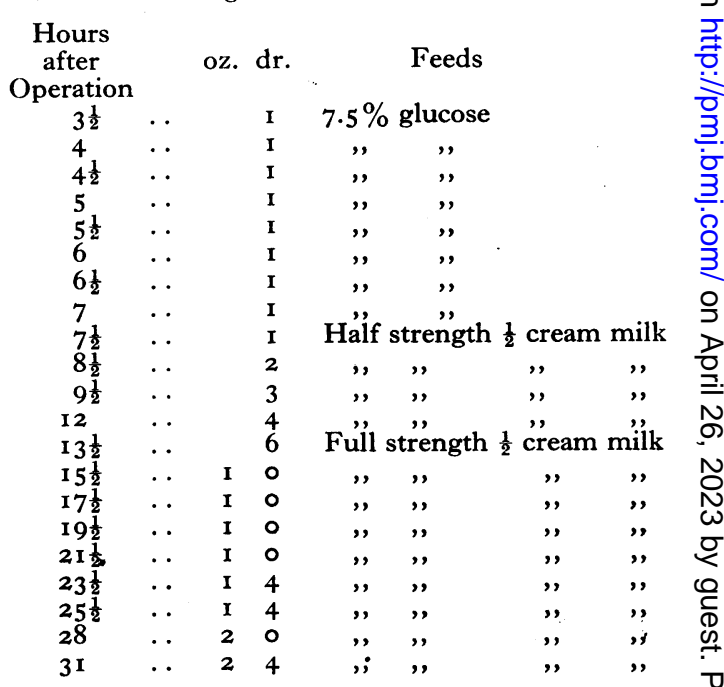

Thereafter three-hourly feeds of 3 oz., increasing gradually according to the child's weight.

Small quantities of water allowed between feeds. 
breast feeding. This is done to minimize the risks of gastro-enteritis to which bottle-fed babies are much more prone. During the author's life, gastro-enteritis has been the cause of the postoperative mortality. Oral streptomycin in association with other medical measures is of value should a bottle-fed child develop diarrhoea.

Appended are post-operative feeding charts which are in use at the present time at the Westminster Children's Hospital.

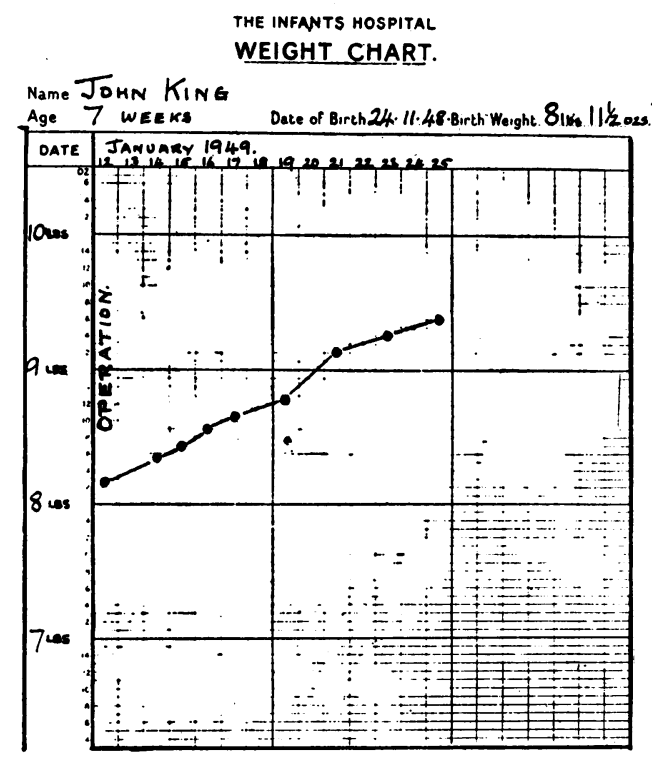

FIG. I.-Typical post-operative weight chart.

Children treated in this way make excellent recoveries. Fig. I is a typical weight chart. Ten years ago the author published a consecutive series of 100 breast-fed babies suffering from pyloric stenosis operated on at the Wesminster Children's Hospital without a death. The war intervened. Since then a further 47 cases have been operated on by him at that hospital without fatality. Of these 47,28 were breast-fed, 19 bottle-fed; 40 were males, 7 females. One case suffered from erythroblastosis foetalis, whilst one case developed gastro-enteritis and a urinary infection after operation.

Between the years 1934 and 1948 , a further 50 cases were operated upon by him at the Luton Children's Hospital; 39 were males, I I females. None of these cases was given fluids pre-operatively except by mouth. All were operated upon on the day the diagnosis was made. There were again no deaths.

\section{CONGENITAL DUODENAL ATRESIA}

Duodenal atresia differs from hypertrophic $\frac{\widehat{D}}{8}$ pyloric stenosis in that it causes symptoms from $c$ birth; vomiting comes on immediately. The $\Rightarrow$ children are apathetic and sleepy.

There are two clinical groups into which these patients fall, the first in which the obstruction is $\bar{F}$ complete, the second in which it is only partial, and $\frac{\bar{\omega}}{\partial}$ two pathological groups, intrinsic and extrinsic. $\stackrel{\odot}{\curvearrowright}$ The intrinsic form is a malformation of the duodenum consisting of either (I) a complete discontinuity of the bowel, (2) an atresia, the two. ends of the duodenum being linked by a fibrous $\vec{\omega}$ cord which may or may not have a small lumen, or o (3) the presence of a septum across the duodenum.

These malformations may occur either proximal 3 or distal to the bile papilla. If proximal, the vomitus contains no bile; if distal, it is bile- i stained. The latter, distal obstruction, is commoner in the proportion of $5: 1$.

Extrinsic obstruction is the gut, being produced by a volvulus, or by peritoneal bands and adhesions resulting from the $\vec{c}$ associated plastic peritoneal reaction.

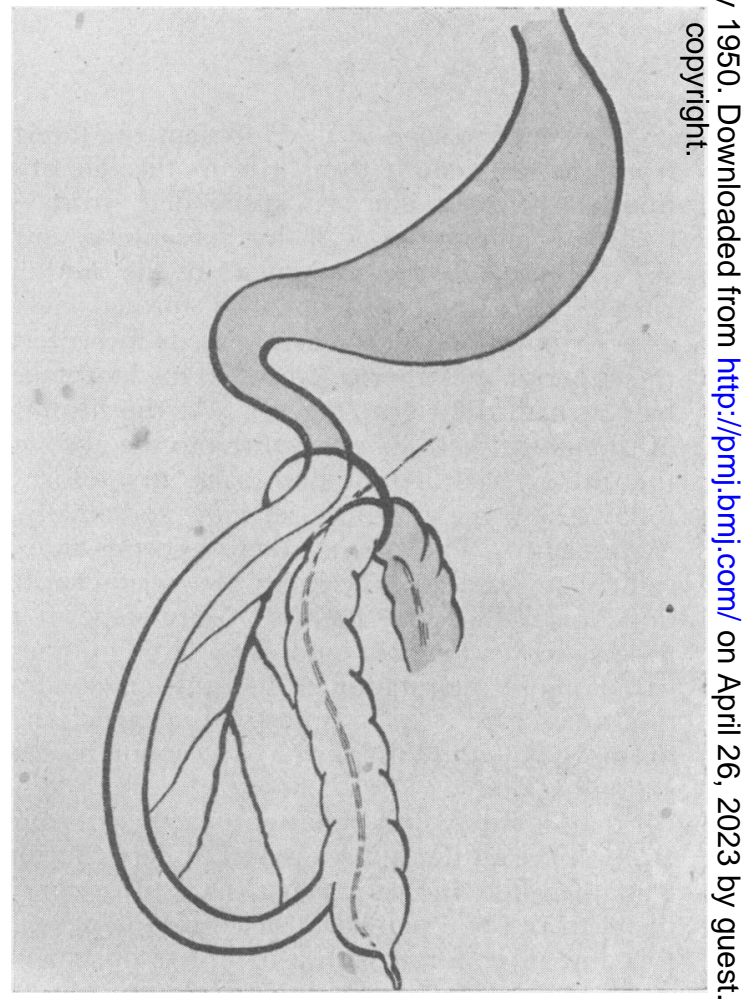

Fig. 2. - The intestine from duodenum to midtransverse colon lies outside the abdomen in the umbilical cord. 


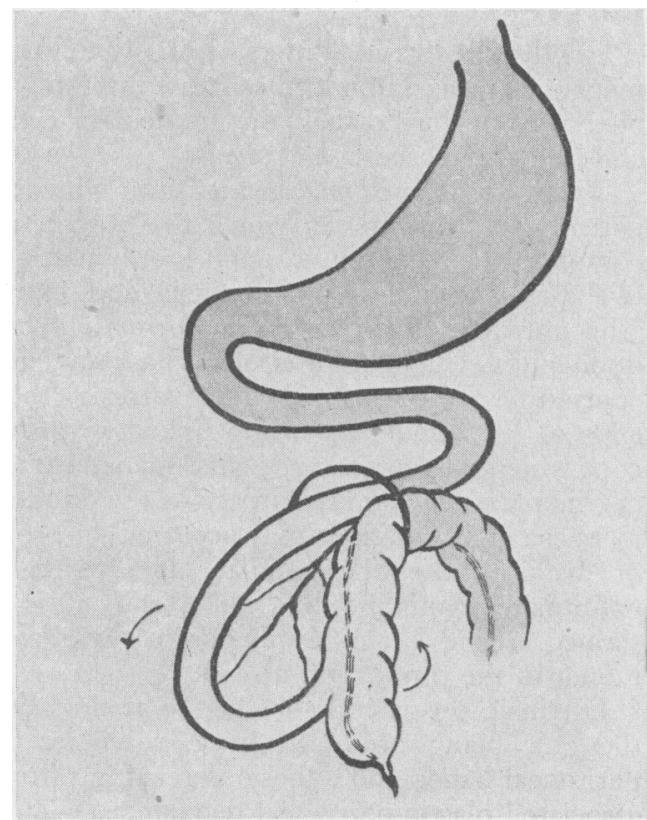

FIG. 3.-The loop takes a half turn anti-clockwise.

At an early period of development the intestine from the duodenum to a little to the left of the mid-transverse colon is suspended from the posterior abdominal wall by mesentery and is situated outside the abdomen in the umbilical cord (Fig. 2). The loop thus formed has an artery running down the centre of its mesenterythe superior mesenteric artery. This loop takes a half turn to the right (Fig. 3). At the beginning of the tenth week the gut returns to the abdomen, the pre-arterial loop going back first (Fig. 4), followed by the post-arterial loop and finally by the caecum. The caecum then descends into the right iliac fossa and the areas of "peritoneal fixation' fuse (Fig. 5). Failure at any stage of this process leaves the way open for a large number of variations of malrotation of the gut, and malrotation may give rise to intestinal obstruction by means of volvulus, kinking or obstruction by bands and adhesions.

Such infants vomit persistently soon after birth. In both the author's cases reported here the vomit contained bile, indicating that the obstruction was distal to the bile papilla. Meconium is passed at first but in children with a complete obstruction, stools soon cease except for a little mucus. Dehydration and loss of weight supervene and death occurs in about ten days.

If the obstruction is only partial, the infant may

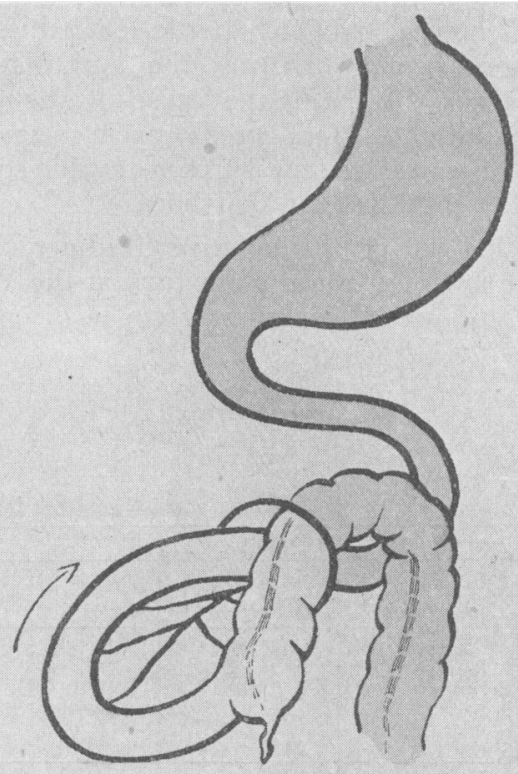

FIG. 4.-At the beginning of the tenth week the gut returns to the abdomen, the pre-arterial loopgoing back first.

survive indefinitely. Physical examination may veal an active stomach but is otherwise negative and unlike in hypertrophic pyloric stenosis, a $\mathrm{X}$-ray examination may be of the greatest valuof No time should be wasted in trying alterations in feeding. The Health Service has put a mobi $\mathrm{X}$-ray plant in reach of every home. It should be used.

Barium need not be given for the gas shadows may show all that is needed. All babies are bor without air in the gastro-intestinal tract, but the soon swallow air which appears first in the stomach (Fig. 6), then in the small gut and finally in the colon (Fig. 7). This march of the air which occurs within the first few hours of life is of great diagnostic value and all babies who vomit at birth should be X-rayed. Often this one examinatiof will pinpoint the site of the intestinal obstruction. (Fig. 8). The diagnosis can be confirmed by using a contrast medium, but if barium is used and the lesion is in the small gut, the inspissateg barium in the gut may present a problem and mago in itself produce obstruction. A lipiodol meal preferable.

Operation should be undertaken as soon as the diagnosis is established.

The procedure in duodenal atresia is the re verse of that in pyloric stenosis. A genero anaesthetic is essential in order to discover the fud 


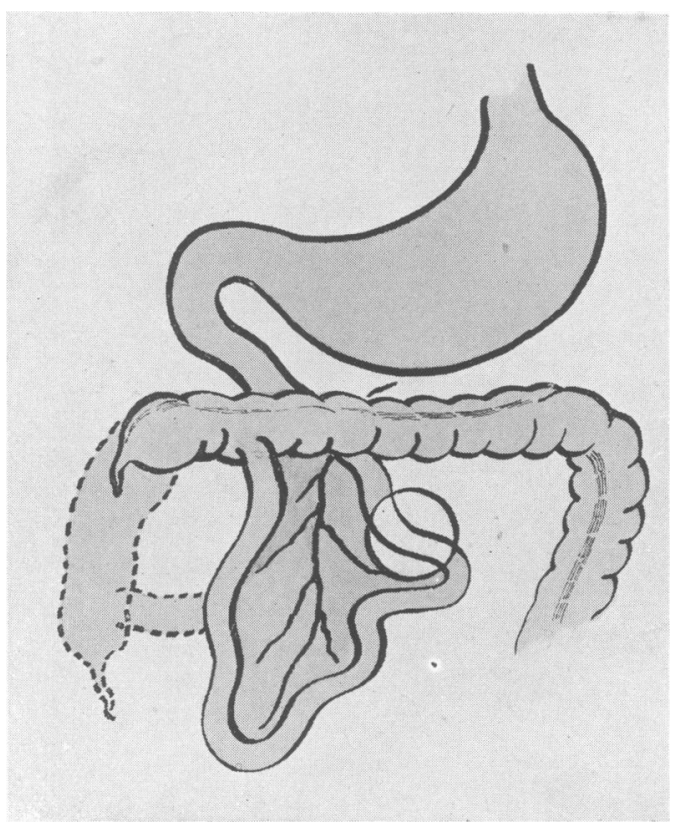

FIG. 5-The caecum finally descends into the right iliac fossa.

extent of the abnormalities present. To do this, it may be necessary to eviscerate the child, which can only be done under full anaesthesia. In the author's experience these children stand evisceration well, shock being apparently less the younger the infant.

In a warm theatre the child is placed on hot water bottles filled with water at blood heat. The umbilical stump looks septic but is apparently worse in appearance than in fact. Through an ample incision the viscera are delivered on to the abdominal wall in order to discover accurately the nature of the congenital abnormality. A malrotation of the gut is then corrected, whilst if the obstruction is due to a band, it is divided. Sometimes two lesions may co-exist. It may be necessary to cut the peritoneum on the lateral side of the second part of the duodenum in order to free it and follow it round. After a volvulus has been reduced, there may still be a drag on the duodenum (Fig. 9).

\section{Case I}

H.S. Admitted, September 8, I947, aged 16 days. Full term birth, weight, $7 \mathrm{lb} .7 \mathrm{oz}$.

History of melaena from second to fifth days.

Since birth vomited bile-stained fluid one to one and a half hours after food. Weight on admission, $6 \mathrm{lb}$. I oz.

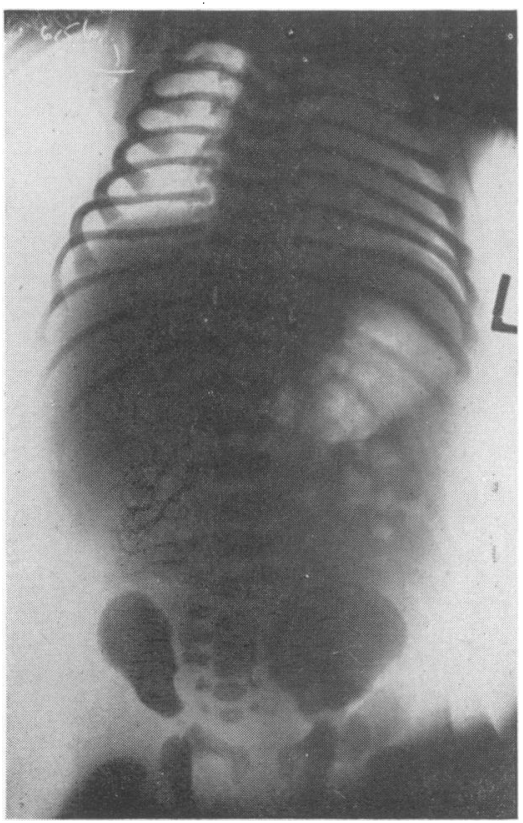

Fig. 6.-Swallowed air in the stomach and upper small intestine in a normal child 1 hour and 40 minutes after birth.

Barium Meal. No barium passed beyond? second part of duodenum in one hour.

Laparotomy on day of admission, after gastric lavage.

General anaesthesia. Duodenum found to be obstructed by two thick bands in position AA (Fig. 9). Duodenum appeared to be patent.

Malrotation of gut present. The child passed bile-stained stools six days after operation, but 12 days after operation developed loose stools and vomiting and died a week later.

Post-Mortem Examination. Wasted, dehydrated female infant. Well-healed upper abdominal vertical incision. Some patchy collapse of the lungs. Thorax otherwise normal.

Abdomen. No excess fluid. Left lobe of liver adherent to scar in abdominal wall. Numerous adhesions round site of operation. Great omentum absent.

Stomach. Distended, otherwise normal. Pylorus normal.

Duodenum. First and second parts distended. Mucosa of duodenum and upper inch or two of ileum appeared hyperaemic. Bile entered normally on pressure on gall bladder. Duodenum compressed by band consisting of aberrant first part of colon and root of mesentery (Fig. 9).

Small Intestine. The root of the mesentery 


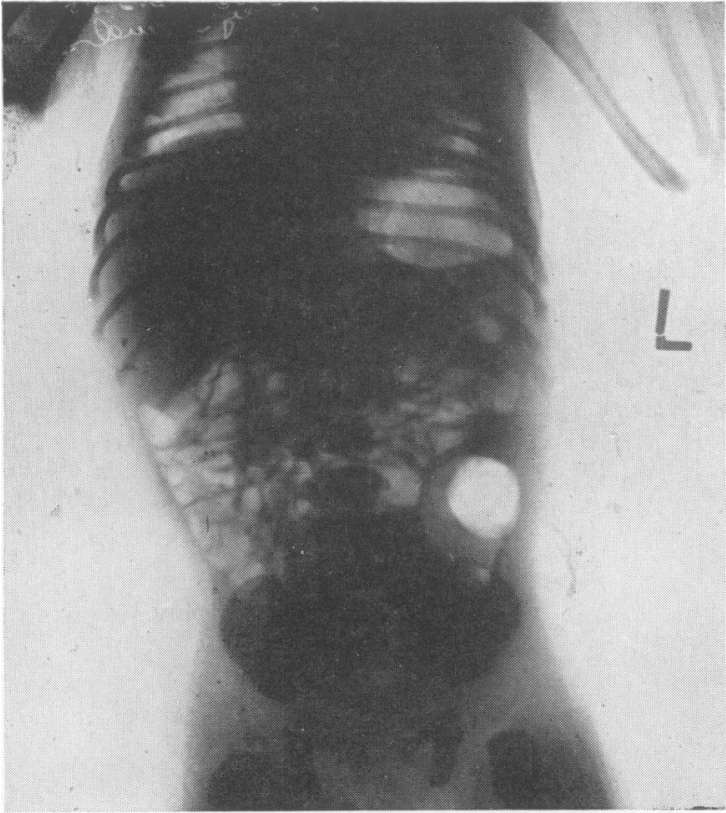

FIG. 7.-Swallowed air in the stomach, small intestine and colon in a normal child to hours after birth.

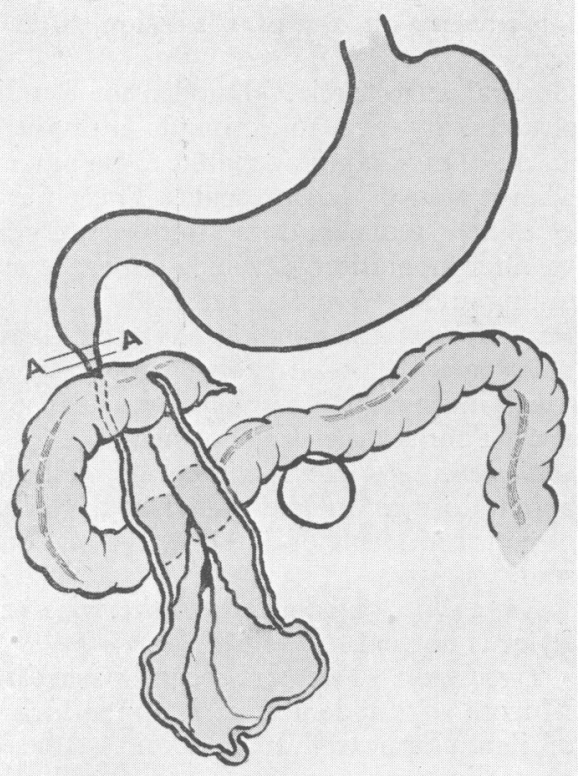

Fig. 9.-Case I. Malrotation of the bowel with obstruction of the second part of the duodenum, caused by two thick bands in the position AA.

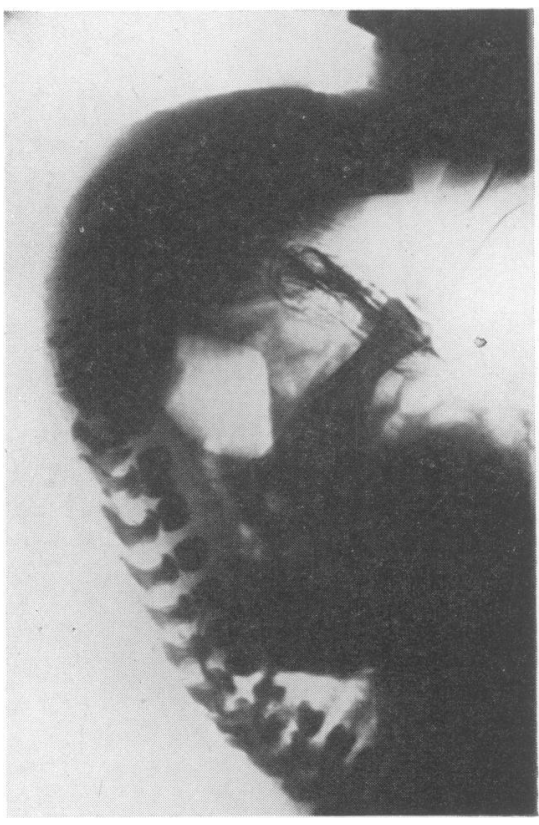

FIG. 8.- Infant held upside down, showing air in the bladder and rectum which communicate. Diag- $\vec{\varphi}$ nosis: Rectovesical fistula with imperforate anwer. O

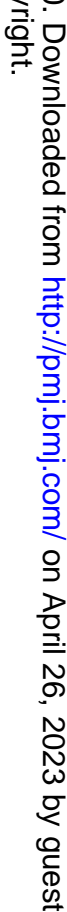

FIG. I0.- Straight $\mathrm{X}$-ray of abdomen in a case of atresia of the second part of the duodenum, show-을 ing gross distension of the stomach and duodenum $\mathbb{D}$ with air. None has passed on into the distal bowel. $\frac{\mathrm{C}}{\mathbb{D}}$ 


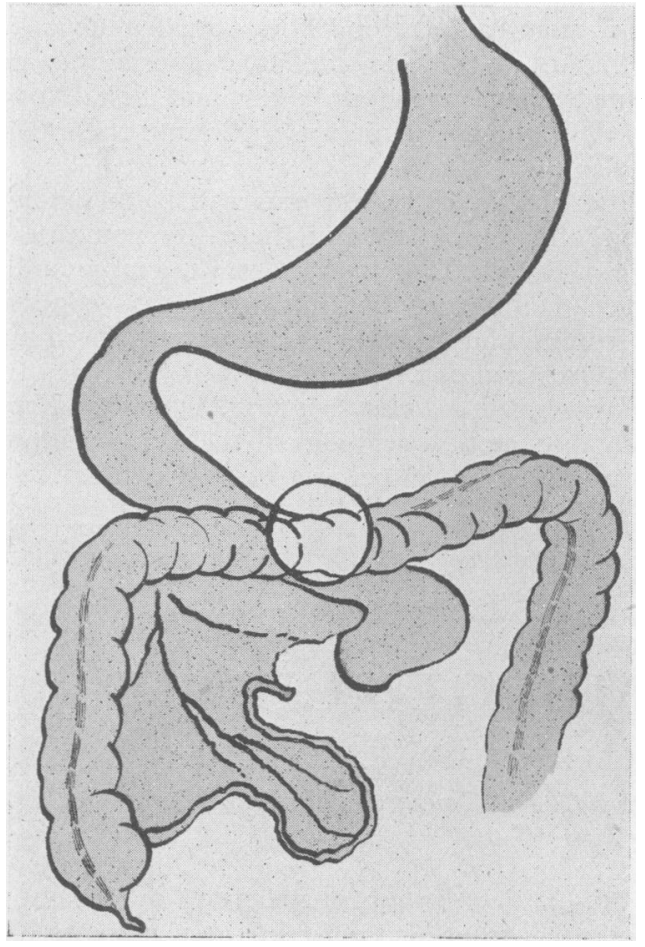

FIG. II.-Case of congenital absence of a part of the upper jejunum, with gross distension of the stomach and duodenum above.

was superior to the first part of colon. Otherwise normal. Contents bile stained. No collapse or distension.

Large Intestine. Ileo-colic junction lay just below the pyloric end of stomach. Appendix normal. First part of colon then ran behind the root of the mesentery and thereafter ran beneath the stomach to a normal splenic flexure. Descending colon normal.

Rest of abdomen normal.

Summary. Malrotation of intestine. Enteritis. In cases of intrinsic obstruction of the duodenum, either partial or complete, a gastroenterostomy should be performed. This may be either anterior or posterior, depending upon the condition found and the way in which the jejunum lies most easily. A clamp may be used on the stomach but not on the intestine, which is very small. Finest embroidery needles threaded with silk are used. For suturing the stomach to the jejunum, various aids to suture have been described such as the passage of a metal-tipped tube into the stomach, and the distension of the collapsed small gut with air injected with a syringe. The author has not found them of value.

\section{Post-Operative Treatment}

The child is put back to bed and is given intravenous glucose 4.3 per cent. in saline o. 8 per cent. and the drip is continued until the child can safely take fluids by mouth. A tube is put into the stomach and continuous gastric suction is established for 48 to 56 hours. Feeding is then commenced. The stomach contents are aspirated at first before each feed to prevent overdistension of the stomach. When gastric function is reestablished the drip is discontinued.

The importance of an intravenous drip in these cases cannot be overstressed. By its means the hydration and, to a large extent, the nutrition of the child, can be maintained during the critical period before the gastro-enterostomy begins to function.

The author has previously reported two patients successfully operated on in this way (Proc. Roy. Soc. Med., 1935, 29, 1213; Ibid., 1938, 31, 761). The latter child is now I I years old, in good health and at a preparatory school. A further recent case is appended:-

\section{Case 2}

J.C. Born, July 5, I948, weighing $6 \mathrm{lb} .3 \frac{3}{4} \mathrm{oz}$.

Vomited bile-stained fluid from birth.

First seen, July 20, I948. Weight, 5 lb. $6 \frac{1}{2}$ oze Slight icteric tinge.

Barium Meal. No barium passed into the jejunum in $\mathrm{r} \frac{1}{2}$ hours (Fig. IO).

Laparotomy same day, after stomach wash out.

First and second parts of duodenum found to be enormously distended. Atresia of the second part beyond the biliary papilla. Whole of small gut collapsed and very small.

Posterior gastro-enterostomy performed. On return to ward an intravenous drip and continuous gastric suction through a small catheter set up. This treatment was continued until July 23 , when fluids were started by mouth. Drip discontinued, July 24. Breast feeding was resumed and progress was satisfactory. The child was discharged on August 30 , having regained its birth weight. It was last seen on March 21, 1949, in good health and weighing $22 \mathrm{lb}$. I oz.

\section{Case 3} days.

L.S. Admitted January 22, I949, aged four

No stool since birth. Had taken small feeds only which it vomited promptly. Normal pregnancy; full-term delivery.

Examination showed a dehydrated baby, thought to be slightly jaundiced. The abdomen was distended, especially in the upper part; the lower part was dull to percussion. No mass felt. The child vomited bright yellow, mucus-containing 
fluid and proceeded to pass its first stool, consisting of opaque gelatinous ovoids with a few darker pellets, slightly bloodstained.

Straight $X$-ray of the abdomen showed the presence of gas in the stomach, but none elsewhere in the bowel.

Operation the same day under local anaesthesia later supplemented with cyclopropane and oxygen. Grossly dilated small bowel was delivered and followed to a blind end, where there was a gap of several inches before collapsed gut began. The mesentery was present opposite the undeveloped segment but was very thin. The proximal loop was emptied by putting a catheter into it and a large quantity of thin, bile-stained mucus was? aspirated. A side-to-side anastomosis was per-ه formed between the proximal and distal loops:$90 \mathrm{ml}$. of glucose saline was given into the internal jugular vein.

The child's condition was satisfactory for 36 hours after operation, but thereafter vomiting re curred, the stomach aspirations were large and, inf spite of intravenous infusions, its condition deteriorated and death occurred on the fourthe post-operative day.

Post-mortem examination (Fig. .I I) showed com- $\overrightarrow{0}$ plete absence of a segment of the upper jejunum $\overrightarrow{\vec{t}}$ the proximal bowel being grossly distended.

The author wishes to thank the physicians of the Westminster Children's Hospital for permission to publish cases referrea by them; and Mr. Albert Davis for his kindness in obtaining $X$-rays showing gas shadows in the intestines of newborn infants

\title{
ANAESTHETIC EMERGENCIES
}

\author{
By J. Alfred LeE, M.R.C.S., L.R.C.P., M.M.S.A., D.A., F.F.A., R.C.S. \\ Consultant Anaesthetist to The General Hospital, Southend; The General Hospital; Rochford; \\ King George Hospital, Ilford, etc.
}

Emergencies are always hiding round the corner, waiting to pounce on the anaesthetized patient, and it is axiomatic that throughout the whole of the administration the anaesthetist should be intelligently alert. The price of safety is constant watchfulness - in somno securitas.

Anaesthetic emergencies cannot always be treated successfully; and it is a melancholy reflection that deaths under anaesthesia are not decreasing. When calculated on a quarter of a million cases from five teaching hospitals in three countries, the death rate was about one in a thousand (Gillespie, 1944). The operating theatre is no place for a display of virtuosity, and it is always wiser to use an agent or method with which one is familiar than to employ a technique with which one is not au fait, just because it may be theoretically indicated.

Crises can arise in the anaesthetic room, in the operating theatre or during the immediate postoperative period.

\section{Anaesthetic Room Emergencies}

It will occasionally happen that a patient arrives for operation in a state of depression consequent on the ill-judged dosage of sedative premedication. The writer has known operations postponed for this reason. Now and again opium derivatives seem to exert an overwhelming effect even if the dosage is appropriate, and the patient may be breathing no more than six or eight times a minute. Bromethol, likewise a respiratory depressant, may seriously reduce the rate and depth of breathing.
If minor degrees of obstruction of the upper respiratory tract also exist in these patients, thoyco may soon become quite seriously hypoxic, and 4 fo they are in addition physically handicapped, state of emergency may soon develop.

The remedy here is partly preventive and partly curative. The dosage of powerful drugs should bea ' tailor made' to suit the type and constitution of each individual patient. It should be remembered that old people, those suffering from chronic de-o윽 bilitating disease, those of hypothyroid constitution and those already receiving sedative drugs, wille probably require less opiate or barbiturate than the average patient. The modern treatment of Graves' disease may produce in the thyrotoxic: patient a temporary hypothyroidism which will 3 make it difficult for him to deal with doses of bromethol normally suitable for such patients? before operation. When these depressed patients are transferred to the care of the anaesthetist, their condition should improve. He is able to ad $-\frac{7}{0}$ minister mixtures rich in oxygen, secure a good airway and make a smooth induction. He should,$N$ however, bear in mind that if he uses moro respiratory depressants during anaesthesia he must be prepared to assist the normal respiratory effort of the patient.

Scopolamine hydrobromide is a useful drug for two reasons; it reduces secretions from the upperes respiratory tract and it produces amnesia. It should not be used as a routine, however, in patients over 60 years of age, because occasionally a state of restlessness or even delirium may 